\title{
Análise dos fatores que motivam os pacientes adultos a buscarem o tratamento ortodôntico
}

\author{
Liliana Ávila Maltagliati*, Luciana Andrade do Prado Montes**
}

\begin{abstract}
Resumo
Objetivo: este artigo teve como objetivo conhecer os principais fatores que motivam os pacientes adultos a buscarem o tratamento ortodôntico. Metodologia: 70 pacientes adultos (44 mulheres e 26 homens) que compuseram a amostra preencheram questionários contendo uma lista de condições onde eles pudessem identificar suas queixas e motivações para o tratamento. Estatísticas descritivas foram obtidas dos questionários respondidos. Resultados e Conclusões: os resultados demonstraram que a estética relacionada ao posicionamento dentário envolvendo os incisivos superiores foi o fator que exerceu maior influência sobre a motivação dos pacientes adultos a buscarem o tratamento ortodôntico; houve pouca percepção das anomalias esqueléticas por parte dos pacientes entrevistados ( $7 \%$ da amostra) e a sintomatologia dolorosa apareceu como o segundo maior fator motivador.
\end{abstract}

Palavras-chave: Ortodontia. Adulto. Motivação. Estética.

\section{INTRODUÇÃO}

A aparência facial exerce um importante papel no julgamento da atratividade pessoal e também no desenvolvimento da auto-estima ${ }^{17}$. A percepção da aparência, principalmente da face, afeta a saúde mental e o comportamento social com implicações significativas nas áreas da educação e profissional, bem como na vida afetiva (BERSCHEID apud GRABER, VANARSDALL ${ }^{4}$ ). No entanto, alguns estudos têm demonstrado que a percepção da própria aparência envolve inúmeros aspectos que não necessariamente estão relacionados com a real intensidade de deformidade facial ou, mais especificamente, com a gravidade da má oclusão apresentada ${ }^{10,17}$. Irregularidades menores, como um apinhamento ântero-inferior suave ou um diastema mediano, podem chamar mais aten- ção e incomodar muito mais o paciente do que anomalias mais graves, como excesso vertical de maxila, prognatismo ou assimetrias ${ }^{3}$.

A preocupação com a saúde e a estética faciais, que inclui obviamente a saúde e estética dentárias, se altera de acordo com a idade e com as condições socioculturais ${ }^{15}$ e parece haver uma motivação maior do gênero feminino para se submeter ao tratamento ortodôntico, em relação ao masculino ${ }^{14,17,18}$.

As mulheres demonstram ser mais exigentes com a própria aparência, apresentando maior nível de insatisfação e, conseqüentemente, estão presentes em maioria na amostras avaliadas ${ }^{5,7}$. Os indivíduos solteiros e que possuíam formação universitária também foram maioria dentre os avaliados ${ }^{14}$.

Segundo Sergl e Zentner ${ }^{14}$, dois fatores-chave

* Professora Titular da UMESP Coordenadora do Curso de Especialização em Ortodontia da UMESP.

** Mestre em Ortodontia pela Universidade Metodista de São Paulo - UMESP. 
parecem exercer influência significativa na decisão dos adultos em submeterem-se ao tratamento ortodôntico. $\mathrm{O}$ primeiro está na dependência da quantidade e da qualidade das informações a respeito do tratamento e na capacidade de apropriarse dessas informações e transformá-las em decisão. $\mathrm{O}$ segundo fator diz respeito à motivação do paciente, que está intimamente relacionada com a percepção da deficiência da própria aparência estética e que atinge com maior freqüência mulheres e pessoas que trabalham com o público.

No trabalho de Hamdan ${ }^{5}, 40 \%$ da amostra declararam sentir-se desconfortáveis com a própria má oclusão (71\% mulheres e 29\% homens) e 93\% da amostra total buscaram o tratamento principalmente por razões estéticas. Um quinto dos pacientes da amostra relatou uma segunda razão após a estética, entre elas a dificuldade mastigatória e os problemas articulares $(11 \%)$ e as dificuldades na fala (7\%). Hamdan ${ }^{5}$ também observou que houve uma fraca correlação entre o descontentamento com a própria oclusão e o índice de necessidade de tratamento (IOTN), sugerindo que a severidade da má oclusão, provavelmente, exerce um papel menos importante, na decisão da busca pelo tratamento, do que poderíamos supor. Outros fatores, como o nível socioeconômico e a percepção individual dos benefícios psicológicos, possivelmente, exercem maior influência. A satisfação com o progresso do tratamento dos filhos também demonstrou funcionar como um estímulo adicional para que os pais buscassem o tratamento ortodôntico. Neste mesmo trabalho, quase $70 \%$ da amostra reconheceram sentir desconforto em relação à própria aparência, sendo que para $30 \%$ esta insatisfação chegava a provocar grande insegurança, a ponto de estabelecer nítidas desvantagens sociais.

A mensuração do grau de severidade de uma má oclusão e a necessidade de tratamento é difícil e sujeita à diversidade de opiniões e conceitos ortodônticos que se confundem com a queixa principal do paciente. Um método bastante útil e utilizado é o índice de necessidade de tratamento (IOTN), que define a severidade dos desvios oclusais que possam ameaçar a longevidade da oclusão. Este índice possui um componente dentário (DHC) e um componente estético (AC) com validade e confiabilidade verificadas por vários autores ${ }^{5,7,15,16}$. Também tem recebido algumas modificações, no intuito de assegurar maior fidelidade aos resultados, especialmente quando utilizado em pesquisas realizadas por dentistas não especialistas (clínicos-gerais). Entretanto, esses índices oclusais são eficientes para definir a necessidade de tratamento somente do ponto de vista clínico, sem tecer considerações sobre a percepção funcional, estética e psicológica do paciente, sobre a necessidade de tratamento que o mesmo acredita ter $^{5}$, tão importante no norteamento do planejamento, principalmente de pacientes adultos ${ }^{8}$.

É comum deparar-se com pacientes que não fazem idéia sobre a variedade de recursos atuais disponíveis e a amplitude das mudanças faciais que podem acontecer em conseqüência do tratamento ortodôntico/cirúrgico.

Desde as primeiras consultas, tanto o ortodontista como o cirurgião precisam empenhar-se em conhecer profundamente as expectativas dos pacientes em relação ao tratamento, visando adequá-las às reais possibilidades terapêuticas. Este cuidado preliminar aumenta consideravelmente as chances de satisfação com os resultados finais, uma vez que, desde o início, direciona o profissional e o paciente para objetivos factíveis de tratamento. Alguns estudos têm sido realizados com o intuito de colher subsídios para definir o perfil psicossocial dos indivíduos que buscam o tratamento ortodôntico. De acordo com o trabalho de Sergl e Zentner ${ }^{14}, 70 \%$ dos pacientes que ficaram satisfeitos com os resultados obtidos consideraram-se bem informados sobre o tratamento a que estavam sendo submetidos.

Portanto, o tempo despendido para conhecer os verdadeiros interesses do paciente é precioso, sendo de extrema importância informar - da ma- 
neira mais clara possivel - os objetivos, possibilidades e limites do tratamento. Vale considerar que, do ponto de vista do paciente, o tratamento de sucesso é aquele que alcançou as suas expectativas. Sahm et al..$^{13}$ e Mehra et al. ${ }^{10}$ acreditam que a melhor forma de conquistar a confiança e obter a colaboração dos pacientes é dando importância às relações interpessoais e, conseqüentemente, investindo na relação profissional-paciente. Incentivar a participação do paciente na escolha da melhor alternativa de tratamento irá, obrigatoriamente, fazer com que ele reflita melhor sobre as suas reais intenções para com o tratamento, tornando-o co-responsável, e isto refletirá positivamente na relação profissional/paciente.

Tem ficado subentendido na literatura que, para a maioria dos pacientes, o fator motivador de maior impacto psicológico é a percepção individual da própria anomalia e não a opinião e reação das outras pessoas diante do problema ${ }^{3}$. Entretanto, é importante que este tipo de paciente seja muito bem informado sobre as limitações do tratamento ortodôntico, pois a expectativa exagerada aumentará igualmente a sua exigência e a potencial insatisfação com os resultados obtidos. Para Graber e Vanarsdal1 $1^{4}$, o clínico é normalmente despreparado para avaliar psicologicamente o paciente e dificilmente obterá sucesso quando o paciente não apresentar condições psicológicas favoráveis.

Visando encontrar uma forma de facilitar a comunicação entre paciente e profissional, Martins et al. ${ }^{9}$ propuseram a utilização de um questionário, claro e objetivo, apresentando um conjunto de problemas que pudessem ser apontados pelo paciente para definir suas queixas. Por meio deste questionário, avaliaram a concordância existente entre o plano de tratamento proposto pelos ortodontistas e a queixa apresentada pelos pacientes. Os resultados demonstraram que houve um nível de concordância excelente em $64,5 \%$ da amostra, enquanto em $29 \%$ a concordância foi de razoável a boa.

O presente trabalho foi realizado com o obje- tivo de procurar conhecer melhor os anseios e as expectativas dos pacientes no momento em que decidem se submeter ao tratamento ortodôntico. Utilizando um questionário que foi aplicado na consulta inicial e respondido pelo próprio paciente, foi proposto também observar-se quais as queixas mais comuns, os benefícios de tratamento mais conhecidos pelos pacientes e aqueles que são menos conhecidos ou até desconhecidos por eles. Considera-se que a determinação de um método eficiente para obtenção dessas informações representa uma ferramenta importante na interação do profissional com o paciente e, conseqüentemente, no sucesso do tratamento.

\section{MATERIAL E MÉTODOS}

Para a realização deste trabalho, logo na primeira consulta, foi apresentado aos pacientes um questionário, o mesmo proposto por Arnett e Worley ${ }^{1}$ em seu estudo, contendo uma lista de condições para que eles pudessem identificar suas queixas (Quadro 1). O conteúdo do questionário foi dividido em 3 categorias: 1) modificações dentárias, 2) modificações faciais e 3) modificações dos sintomas; pois, de acordo com Arnett e Wor1 ley ${ }^{1}$, os pacientes que procuram tratamento ortodôntico, geralmente, estão em busca de mudanças em alguma destas categorias. O questionário foi respondido pelos próprios pacientes, possibilitando que eles identificassem suas queixas e também tivessem a oportunidade de especificar em que sentido deveria ser feita a correção. Desta forma, poderia ser identificado o nível de percepção que os pacientes possuiam em relação às suas próprias anormalidades faciais e dentárias.

A amostra foi composta por 70 pacientes (44 mulheres e 26 homens), com idades variando entre 20 e 55 anos, escolhidos aleatoriamente, entre os inscritos em busca de tratamento ortodôntico, nos cursos de Pós-graduação em Ortodontia da Universidade Metodista de São Paulo (UMESP) e que tinham buscado o tratamento por iniciativa própria, sem a indicação de outro profissional. 


\section{Paciente:}

Idade:

Escolaridade:

\section{Motivação do paciente para tratamento ortodôntico}

Você, que decidiu se submeter a um tratamento ortodôntico, provavelmente está buscando mudanças em seus dentes, na sua face ou o alívio de dores ou desconfortos.

Por favor, ajude-nos a entender melhor o seu problema e suas expectativas, marcando com um " $x$ " os itens abaixo. Para ser mais específico faça um círculo em volta das palavras: superiores, inferiores, mais, menos, para a frente, para trás, mais longos, mais curtos, etc.

Dentes

Se seus dentes pudessem ser mudados, como você gostaria de mudá-los?

() Arrumar os dentes da frente superiores/inferiores

() Arrumar os dentes de trás superiores /inferiores

() Tornar os dentes superiores anteriores mais longos / mais curtos

() Movimentar os dentes superiores para frente / para trás

() Movimentar os dentes inferiores para frente / para trás

() Posicionar a linha dos meus dentes anteriores superiores mais para frente/ para trás

() Mover a linha média dentária superior/inferior para direita/ esquerda

Face

Se sua aparência facial pudesse ser mudada, o que você gostaria de mudar?

() Eliminar o queixo duplo

() Movimentar o queixo para a frente/ para trás

() Movimentar o queixo à esquerda/ à direita, para centralizá-lo

() Movimentar o lábio superior para a frente/ para trás

() Movimentar o lábio inferior para a frente/ para trás

() Movimentar a área em torno do meu nariz para a frente/ para trás

() Tornar o perfil do meu nariz mais longo/ mais curto

() Movimentar a área sob os meus olhos para a frente/ para trás

() Tornar minhas bochechas maiores/ menores

() Mostrar mais/menos os meus dentes/ gengivas, quando sorrio

() Tornar meus lábios mais próximos/mais afastados, quando meus dentes estiverem em contato

() Reduzir a tensão em meu queixo/ lábio, quando fecho os lábios

() Tornar minha face mais estreita/ larga

() Diminuir o volume da minha mandíbula na porção inferior lateral da face

Sintomas

Se você sente dor ou desconforto onde ele se localiza?

() $\mathrm{Na}$ frente da minha orelha

() Abaixo da minha orelha direita/ esquerda

() Acima da minha orelha direita/ esquerda

() Em minha orelha direita/ esquerda

() Em meu pescoço do lado direito/ esquerdo

() Em meu ombro direito/ esquerdo

() Em minha têmpora direita/ esquerda

() Em meus dentes

() Em meus seios maxilares

() Em meu olho direito/ esquerdo

Quadro 1 - Questionário preenchido pelos pacientes da amostra. 


\section{RESULTADOS}

Os resultados estão dispostos no gráfico 1, representando, em índices percentuais, a distribuição dos principais fatores de motivação dos pacientes adultos para buscarem tratamento ortodôntico.

O maior interesse dos pacientes esteve relacionado com as mudanças no posicionamento dentário, principalmente em relação aos dentes anteriores (mencionado por $81 \%$ da amostra como principal meta do tratamento). Entre os dentes anteriores, os superiores foram alvo de maiores preocupações por parte dos entrevistados e a queixa em relação aos dentes posteriores apareceu em apenas $24 \%$ da amostra, demonstrando a maior preocupação com a estética, em detrimento da função mastigatória.

Dos itens do questionário que forneceram informações a respeito das alterações faciais, o que foi mais percebido pelos pacientes relaciona-se com o sorriso gengival e excesso de exposição dos incisivos (17\%). Por outro lado, poucos indivíduos mencionaram interesse em fazer com que seus incisivos superiores aparecessem mais durante o sorriso.

A percepção com relação ao deslocamento da mandíbula no sentido sagital e transversal, para

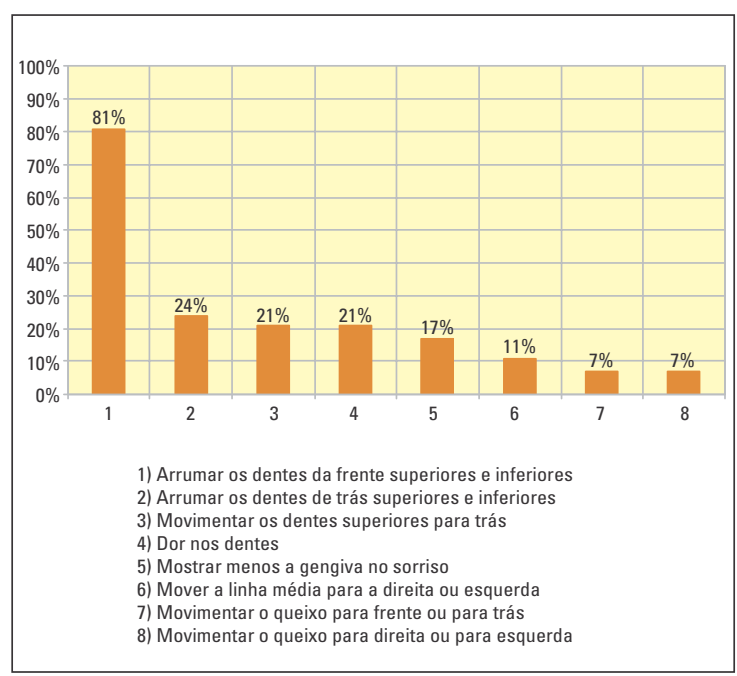

GRÁFICO 1 - Representação gráfica dos principais fatores de motivação dos pacientes adultos para buscarem o tratamento ortodôntico. correção da assimetria facial, foram ambos referidos por apenas $7 \%$ dos indivíduos da amostra. Já a percepção de assimetria de linha média superior foi notada por $11 \%$ dos indivíduos. De importante consideração foi que, no total, $76 \%$ da amostra não apresentaram queixa da aparência facial e isto gerou o questionamento se esta porcentagem vai ao encontro dos casos que são indicados para cirurgia. Mesmo no item relacionado à face, a maior porcentagem de queixa esteve relacionada com os dentes.

Com relação à sintomatologia, a sensibilidade dolorosa nos dentes apareceu como a queixa mais freqüente $(21 \%)$, provavelmente relacionada com o hábito parafuncional de apertamento dentário e/ou bruxismo. Em segundo lugar, apareceram as queixas na região das orelhas e do pescoço.

\section{DISCUSSÃO}

Apesar da amostra ter sido construída aleatoriamente, a quantidade de indivíduos do gênero feminino foi quase o dobro em relação à do gênero masculino, reforçando os achados de Tuominen et al. ${ }^{18}$, Sergl, Zentner ${ }^{14}$; Tung e Kiyaki ${ }^{17}$, que demonstram um maior interesse e disposição do gênero feminino em se submeter ao tratamento ortodôntico. O fato dos homens estarem em menor número na amostra pode estar relacionado com uma menor exigência masculina em relação à própria imagem, como também a uma menor disposição em aceitar as regras do tratamento.

A percepção das anomalias esqueléticas ficou em torno de apenas $7 \%$, o que está de acordo com os resultados do estudo de Gosney ${ }^{3}$, que revelaram que os pacientes podem conviver bem com anomalias muito graves e por outro lado se sentirem extremamente incomodados com irregularidades menores, como dentes desalinhados ou até mesmo pequenos diastemas. Portanto, o paciente adulto, geralmente, não percebe ou não tem queixa da aparência facial, mesmo nos casos com discrepância acentuada, onde a indicação seria cirúrgica. Isto pode acontecer por dois motivos: 1) ignorância do 
paciente sobre os atuais recursos disponíveis para promover mudanças no aspecto facial e 2) como as desarmonias faciais são de ordem genética, os indivíduos se reconhecem no seu grupo familiar e isto faz com que eles se aceitem esteticamente.

Em face da característica da estética dentária ter sido sensivelmente superior na queixa dos pacientes adultos, resultado semelhante ao encontrado por Hamdan ${ }^{5}$, deve-se questionar se a percepção do que é realmente necessário alterar está em sintonia com o desejo de mudança do paciente. Talvez o maior desafio do ortodontista esteja em aceitar a queixa do paciente como principal fator a ser corrigido e não ficar aprisionado ao diagnóstico baseado em análises cefalométricas, morfométricas, análise numérica e de modelos ou até mesmo em análises faciais.

Se, por um lado, o uso da cefalometria tirou a liberdade do ortodontista, impedindo-o de enxergar as evidências visuais proporcionadas pelo exame clínico, por outro, mesmo as análises morfológicas e clínicas possuem uma dose de subjetividade e das preferências pessoais de cada profissional, aumentando as chances de elaboração de um plano de tratamento que não seja bem aceito pelo paciente. Talvez o segredo esteja em flexibilizar os conceitos sobre harmonia facial e dentária, priorizando a queixa do paciente e aceitando a não intervenção em desvios passíveis de correção, mas que estejam fisiológicos para oclusão, uma vez que estejam permitindo a manutenção da saúde oclusal e periodontal ${ }^{8,12}$.

Apesar da sintomatologia dolorosa nos dentes ter aparecido como queixa para $21 \%$ da amostra, a quantidade de indivíduos que mencionava no questionário algum tipo de dor foi de $47 \%$. No entanto, não podemos assegurar que esta dor tenha relação com problemas de âmbito odontológico, mas demonstra o quanto o desconforto estimula a busca por algum tipo de tratamento que possa promover o alívio dos sintomas.

Ao certo, o interesse pelo tratamento ortodôntico tem aumentado nas últimas décadas, com o aumento da expectativa de vida e com as exigências estéticas da sociedade contemporânea, que solicita a longevidade da saúde dentária. Exige-se do profissional discernimento para entender os anseios do paciente, conscientizá-lo das limitações existentes e elaborar o plano de tratamento suficiente para contemplar a queixa principal, delimitando o que realmente necessita de intervenção.

\section{CONCLUSÕES}

De acordo com os resultados obtidos podemos concluir que:

1) A estética relacionada ao posicionamento dentário é o fator que exerce maior influência sobre a motivação dos pacientes adultos a buscarem o tratamento ortodôntico.

2) Houve pouca percepção das anomalias esqueléticas por parte dos pacientes entrevistados (apenas 7\% da amostra).

3) A principal meta de tratamento apontada foi a correção de problemas envolvendo os incisivos superiores.

4) A sintomatologia dolorosa apareceu como o segundo maior fator motivador. 


\title{
Analysis of the factors that induce adult patients to search for orthodontic treatment
}

\begin{abstract}
Aim: The purpose of this article was to determine the main reasons that motivate adult patients to seek for orthodontic treatment. Methods: The sample comprised 70 adult individuals ( 44 females and 26 males) that were asked to answer a questionnaire with a list of possible complaints. Descriptive statistics were obtained. Results and Conclusions: The findings showed that esthetics related to malpositioning of the upper incisors is the reason mostly associated to the search for orthodontic treatment. Only a few patients ( $7 \%$ of the sample) indicated skeletal discrepancies as reason for treatment. Symptoms of TMJ appeared as the second reason.
\end{abstract}

Key words: Orthodontics. Adults. Motivation. Esthetics.

\section{REFERÊNCIAS}

1. ARNETT, G. W.; WORLEY, C. M. The treatment motivation survey: defining patient motivation for treatment. Am. J. Orthod. Dentofacial Orthop., St. Louis, v. 1, no. 15, p. 233-238, 1999.

2. FRITZ, U.; DIEDRICH, P.; WIECHMANN, D. Lingual technique: patients'characteristics motivation and acceptance. J. Orofac. Orthop., München, n. 63, p. 227-233, 2002.

3. GOSNEY, M. B. E. An investigation into some of the factors influencing the desire for orthodontic treatment. Br. J. Orthod. Oxford, v. 13, no. 2, p. 87-94, Apr. 1996

4. GRABER, T. M.; VANARSDALL, R. L. Ortodontia: princípios e técnicas atuais. 3. ed. Rio de Janeiro: Guanabara Koogan 2000

5. HAMDAN, A. M. The relationship between patient, parent and clinician percieved need and normative orthodontic treatment need. Eur. J. Orthod., Oxford, v. 26, no. 3, p. 265-271, 2004

6. KIYAK, H. A. McNEILL, W. WEST, R. A. The emotional impact of orthognatic surgery and conventional Orthodontics. Am. J. Orthod., St. Louis, v. 88, no. 3, p. 224-234, 1985.

7. KLAGES, U.; BRUCKNER, A.; ZENTNER, A. Dental aesthetics, self-awareness and oral health-related quality of life in young adults. Eur. J. Orthod., Oxford, v. 26, no. 5, p. 507-514, 2004

8. MALTAGLIATI, L. A. Tratamento ortodôntico em adultos: Considerações psicológicas e de diagnóstico. In: SAKAI, E. et al. Nova visão em Ortodontia-Ortopedia Funcional dos Maxilares. São Paulo: Ed. Santos, 2004. cap. 32, p. $585-590$.

9. MARTINS, L. F.; REIS, S. A. B.; SCANAVINI, M. A.; VIGORITO J. W. Comparação entre o diagnóstico ortodôntico e a expectativa do paciente em relação ao tratamento: proposta de um questionário que facilite a comunicação entre pacientes e profissionais. J. Bras. Ortodon. Ortop. Facial, Curitiba, v. 8, n. 43, p.19-28, jan./fev. 2003

10. MEHRA, T. NANDA, R. S. SINHA, P. K. Orthodontists assessment and management of pacient compliance. Angle Orthod. Appleton, v. 68, no. 2, p. 115-122, 1998

11. MCKIERNAN, E. X.; MCKIERNAN, F.; JONES, M. L. Psychological profiles and motives of adults seeking orthodontic treatment. Int. J. Adult Orthodon. Orthognath. Surg., Chicago v. 7 , no. 3 , p. $187-198,1992$
12. REIS, S. A. B. Abordagens terapêuticas simplificadas no tratamento ortodôntico de pacientes adultos com problemas ocalizados. In: SAKAI, E. et al. Nova visão em OrtodontiaOrtopedia Funcional dos Maxilares. São Paulo: Ed. Santos, 2004. cap. 33, p. 591-596.

13. SAHM, G.; BARTSCH, A.; KOCH, R.; WITT, E. Subjective appraisal of orthodontic practices. An investigation into perceived practice characteristics associated with patient and parent treatment satisfaction. Eur. J. Orthod., Oxford, v. 13, no. 1, p. 15-21, 1991.

14. SERGL, G. H.; ZENTNER, A. Study of psychosocial aspects of adult orthodontic treatment. Int. J. Adult Orthodon. Orthog nath. Surg., Chicago, v. 12, no. 1, p. 17-22, 1997.

15. STENVIK, A.; ESPLEND, L.; BERSET, G. P.; ERIKSEN, H. M. Atitudes to malocclusion among 18 and 35 years old Norwegians. Community Dent. Oral Epidemiol., Copenhagen, v. 24, no. 6, p. 390-393, Dec. 1996

16. STENVIK, A.; ESPLAND, L.; MATHISEN, A. A longitudinal study on subjective and objective orthodontic treatment need. Eur. J. Orthod., Oxford, v. 19, no. 1, p. 85-92, Feb. 1997.

17. TUNG, A. W.; KIYAK, A. Psychological influences on the timing of orthodontic treatment. Am. J. Orthod. Dentofacial Orthop., St. Louis, v. 113, p. 29-39, 1998.

18. TUOMINEN, M. L.; TUOMINEN, R. J.; NYSTROM, M. E. Subjective orthodontic treatment need and percieved dental appearance among young Finnish adults with or without previous orthodontic treatment. Community Dent. Health, London, v. 11, no. 1, p. 155-167, Mar. 1994.

\section{Endereço para correspondência}

Liliana Ávila Maltagliati

Rua do Sacramento, 230

CEP: 09.640-000 - São Bernardo do Campo / SP

E-mail: lilianamaltagliati@hotmail.com 